\title{
On MQS regulation, innovation and market coverage
}

\author{
Emanuele Bacchiega, ${ }^{*}$ Luca Lambertini*,\#,§ Andrea Mantovani* \\ * Department of Economics, University of Bologna \\ Strada Maggiore 45, 40125 Bologna, Italy \\ \# ENCORE, University of Amsterdam \\ Roetersstraat 11, WB1018 Amsterdam, The Netherlands \\ $\S$ Paul Nitze School of Advanced International Studies \\ Johns Hopkins University Bologna Center \\ via Belmeloro 11, 40126 Bologna, Italy \\ bacchieg@spbo.unibo.it, lamberti@spbo.unibo.it,mantovan@spbo.unibo.it
}

November 3, 2006 


\begin{abstract}
We revisit Maxwell's (1998) analysis to show that MQS regulation has no effects on the high-quality firm's incentive to adopt a more efficient technology in a vertically differentiated duopoly with full market coverage and convex costs of quality improvements which are independent of the scale of production.
\end{abstract}

Keywords: MQS, vertical differentiation, market coverage

JEL: L13, L51, O31 


\section{Introduction}

A relatively small literature investigates the relationship between the introduction of a minimum quality standard (MQS) in a vertically differentiated industry and the high-quality firm's incentive to invest in a new technology characterised by a lower marginal cost (Maxwell, 1998; Garella, 2006).

Maxwell (1998), in particular, nests into a tradition dating back to Gabszewicz and Thisse (1979) to model the role of MQS regulation in a duopoly where the market is fully covered by assumption and firms bear convex costs of quality improvement which are unrelated to the scale of production. He claims that (i) the unregulated equilibrium low-quality level is zero, and (ii) the adoption of MQSs reduces the high-quality firm's incentive to adopt a more efficient technology, ultimately involving a welfare loss. The aim of this note is to show that this is not the case because

- full coverage does require a strictly positive low-quality level;

- for any level of the low quality which is compatible with full market coverage, social welfare is decreasing; therefore, the MQS cannot bite;

- as a consequence, the MQS cannot exert any influence on the highquality firm's incentive to adopt a more efficient technology.

\section{The model}

We take into consideration the same model as in Maxwell (1998, p. 356). A continuum of consumers of mass 1 is uniformly distributed over the interval 
$[\underline{\theta}, \bar{\theta}]$ with $\underline{\theta}>0$ and density $\delta=1 /(\bar{\theta}-\underline{\theta}) \cdot{ }^{1}$ A generic consumer identified by a marginal willingness to pay for quality $\theta \in[\underline{\theta}, \bar{\theta}]$ is characterised by the indirect utility function $U=\theta q_{i}-p_{i}$ if the consumer buys, $U=0$ if he/she does not. $q_{i}$ and $p_{i}$ are the quality and price of the product sold by firm $i$. The market is supplied by two firms, $H$ and $L$, that offer vertically differentiated products characterised by quality levels $q_{H}>q_{L}$. Firm $i$ bears a cost function that depends upon both output and quality, $C_{i}=c x_{i}+F\left(q_{i}, \eta\right)$, where $x_{i}$ is the output level, $c>0$ is a constant marginal cost common to both firms, and $\eta>0$ is a parameter rescaling the cost of quality improvement $F\left(q_{i}, \eta\right)$. As to the latter, which may be considered as the R\&D component of total costs, we assume:

$$
F(0, \eta)=0 ; \frac{\partial F(0, \eta)}{\partial q_{i}}=0 ; \frac{\partial F\left(q_{i}, \eta\right)}{\partial q_{i}}>0 ; \frac{\partial F\left(q_{i}, \eta\right)}{\partial \eta}<0 \forall q_{i} \geq 0, \eta>0
$$

We assume that $U=\theta q_{i}-p_{i} \geq 0$ for all $\theta \in[\underline{\theta}, \bar{\theta}]$, i.e., the market is fully covered. Therefore, the demand system is:

$$
x_{H}=\frac{\bar{\theta}-\widehat{\theta}}{\bar{\theta}-\underline{\theta}} ; x_{L}=\frac{\widehat{\theta}-\underline{\theta}}{\bar{\theta}-\underline{\theta}}
$$

where $\widehat{\theta}=\left(p_{H}-p_{L}\right) /\left(q_{H}-q_{L}\right)$ identifies the consumer who is indifferent between purchasing $q_{H}$ and $q_{L}$. Accordingly, firm $i$ 's profit function is $\pi_{i}=$ $\left(p_{i}-c\right) x_{i}-F\left(q_{i}, \eta\right)$.

As usual, firms' strategic interaction takes the form of a two-stage noncooperative game with simultaneous moves in each stage: in the first, firms choose qualities, in the second they compete in prices. The solution concept is the subgame perfect equilibrium by backward induction.

\footnotetext{
${ }^{1}$ These two assumptions concerning the positivity of $\underline{\theta}$ and the density measure are absent in Maxwell's paper but they are strictly needed.
} 


\section{The game}

For a generic quality pair, optimal prices are:

$$
p_{H}^{*}=\frac{\left(q_{H}-q_{L}\right)(2 \bar{\theta}-\underline{\theta})}{3}+c ; p_{L}^{*}=\frac{\left(q_{H}-q_{L}\right)(\bar{\theta}-2 \underline{\theta})}{3}+c .
$$

Note that the above prices determine the following demands:

$$
x_{H}=\frac{2 \bar{\theta}-\underline{\theta}}{3(\bar{\theta}-\underline{\theta})} ; x_{L}=\frac{\bar{\theta}-2 \underline{\theta}}{3(\bar{\theta}-\underline{\theta})}
$$

which are independent of qualities. Therefore, upstream competition in qualities only determines the size of the unit markup $p_{i}-c$ and the development cost $F\left(q_{i}, \eta\right)$, but does not affect the distribution of consumers across firms. Note that the demand system in (4) is indeed meaningful provided that all consumers, and in particular those patronising the low-quality firm, enjoy a non-negative surplus. This aspect of the model is bound to exert some relevant bearings upon the remainder of the analysis. Moreover, from (4), it appears that $\bar{\theta}>2 \underline{\theta}$ is required in order to ensure $x_{L}>0$.

Using (3), we obtain the relevant profit functions at the quality stage:

$\pi_{H}=\frac{\left(q_{H}-q_{L}\right)(2 \bar{\theta}-\underline{\theta})^{2}}{9(\bar{\theta}-\underline{\theta})}-F\left(q_{H}, \eta\right) ; \pi_{L}=\frac{\left(q_{H}-q_{L}\right)(\bar{\theta}-2 \underline{\theta})^{2}}{9(\bar{\theta}-\underline{\theta})}-F\left(q_{L}, \eta\right)$.

The above expressions entail that the present game has the following crucial property:

Lemma 1 Firms' best reply functions at the quality stage are orthogonal.

Proof. Profit functions (5) are additively separable w.r.t. quality levels. As a consequence,

$$
\frac{\partial^{2} \pi_{i}}{\partial q_{i} \partial q_{j}}=0, i \neq j
$$


This proves the Lemma.

Lemma 1 produces a relevant corollary:

Corollary 2 With full market coverage and fixed costs of quality improvements, MQS regulation leaves unaffected the high-quality level.

This result is in contrast with the acquired wisdom whereby introducing an MQS brings about an increase in all quality levels, given that qualities are strategic complements, as, e.g., in Ronnen (1991). This can be attributed to the full coverage assumption, which is the only substantive difference between the present setup and Ronnen's.

Now we turn to the first order conditions at the quality stage. While:

$$
\frac{\partial \pi_{H}}{\partial q_{H}}=\frac{(2 \bar{\theta}-\underline{\theta})^{2}}{9(\bar{\theta}-\underline{\theta})}-\frac{\partial F\left(q_{H}, \eta\right)}{\partial q_{H}}=0
$$

has an internal solution, $\partial \pi_{L} / \partial q_{L}<0$ everywhere, as long as full coverage holds. On this basis, Maxwell claims that $q_{L}^{*}=0$ (see his Proposition 1, p. 357). However, this is inadmissible in that it would entail a negative surplus for any consumer purchasing the low quality. This can be easily ascertained by checking that $p_{L}^{*}>0$ from (3), while the gross surplus $\theta q_{L}=$ 0 in correspondence of $q_{L}=0$. As a consequence, this would violate the assumption of full market coverage, since all those consumers accounting for $x_{L}$ would be actually unwilling to buy.

In order to preserve full market coverage in absence of an economically meaningful internal optimum for $q_{L}$, firm $L$ should supply at least the quality that makes the consumer identified by $\underline{\theta}$ exactly indifferent between purchas- 
ing or not, given $p_{L}^{*}$ :

$$
\underline{\theta} q_{L}-p_{L}^{*} \geq 0 \forall q_{L} \geq \frac{3 c+q_{H}(\bar{\theta}-2 \underline{\theta})}{\bar{\theta}+\underline{\theta}} \forall q_{H}
$$

where

$$
\frac{3 c+q_{H}(\bar{\theta}-2 \underline{\theta})}{\bar{\theta}+\underline{\theta}}<q_{H}
$$

provided that $\underline{\theta} q_{H}>c$ and $\pi_{L}>0$. While the former condition is always true as it amounts to saying that the poorest consumer in the market must be able to buy the high quality at the marginal cost of a unit of output, the latter may or may not hold depending on the relative size of $c$ and $\eta$, given $\bar{\theta}>2 \underline{\theta}$. Provided $\pi_{L}>0$, we are able to claim:

Lemma 3 The maximum level of $\pi_{L}$ compatible with full market coverage attains at

$$
\widehat{q}_{L}=\frac{3 c+q_{H}(\bar{\theta}-2 \underline{\theta})}{\bar{\theta}+\underline{\theta}} .
$$

Proof. Straightforward, since $\partial \pi_{L} / \partial q_{L}<0$ for all $q_{L} \in\left[\widehat{q}_{L}, q_{H}\right) .^{2}$

By Lemma 2 it is clear that if firm $L$ were forced to guarantee market coverage through the provision of a quality at least equal to $\hat{q}_{L}$, it would choose exactly that level as long as profits are non-negative. The point thus is to examine whether a planner would like to regulate the market by setting a MQS above $\hat{q}_{L}$. To make things more explicit, we follow Maxwell (1998, p. 357) in assuming that $F\left(q_{i}, \eta\right)=q_{i}^{2} / \eta$. If so, then $q_{H}^{*}=(2 \bar{\theta}-\underline{\theta})^{2} \eta /[18(\bar{\theta}-\underline{\theta})]$, and

$$
\widehat{q}_{L}=\frac{54 c(\bar{\theta}-\underline{\theta})+\eta(\bar{\theta}-2 \underline{\theta})(2 \bar{\theta}-\underline{\theta})^{2}}{18(\bar{\theta}-\underline{\theta})(\bar{\theta}+\underline{\theta})} .
$$

\footnotetext{
${ }^{2}$ Note that, if $\pi_{L}<0$ in $q_{L}=\widehat{q}_{L}$, firm $L$ could not search for a lower quality level $q_{L 0}$ such that $\pi_{L}=0$, since $\partial \pi_{L} / \partial q_{L}<0$ implies that $q_{L 0}$ would be incompatible with full coverage.
} 
The desirability of a minimum quality standard can be approached in the following terms. Define the social welfare function:

$$
\begin{aligned}
W= & \pi_{L}\left(p_{H}^{*}, p_{L}^{*}, q_{H}^{*}\right)+\pi_{H}\left(p_{H}^{*}, p_{L}^{*}, q_{H}^{*}\right)+ \\
& +\frac{1}{\bar{\theta}-\underline{\theta}}\left[\int_{\underline{\theta}}^{\bar{\theta}}\left(z q_{L}-p_{L}^{*}\right) d z+\int_{\widehat{\theta}}^{\bar{\theta}}\left(z q_{H}^{*}-p_{H}^{*}\right) d z\right] .
\end{aligned}
$$

The regulator directly influences $q_{L}$, given $\left\{p_{H}^{*}, p_{L}^{*}, q_{H}^{*}\right\}$. We first evaluate the derivative:

$$
\frac{\partial W}{\partial q_{L}}=\frac{(\bar{\theta}-2 \underline{\theta})(\bar{\theta}+4 \underline{\theta})}{18(\bar{\theta}-\underline{\theta})}-\frac{2 q_{L}}{\eta}
$$

at $q_{L}=\widehat{q}_{L}$, yielding $\partial W /\left.\partial q_{L}\right|_{\widehat{q}_{L}} \propto-108 c(\bar{\theta}-\underline{\theta})-(\bar{\theta}-2 \underline{\theta})^{2}(7 \bar{\theta}+\underline{\theta}) \eta<0$. Therefore, the regulator does not desire to increase quality, which amounts to saying that an MQS would not bite. Moreover, and more important, this also implies:

Proposition 4 MQS regulation with fixed costs of quality improvement is incompatible with full market coverage.

By setting an MQS equal to the solution of (12), the regulator would violate the assumption of full market coverage upon which prices (3), the high-quality level $q_{H}^{*}$ and the social welfare function (11) are based. Dropping the full coverage assumption would open a different perspective where it is known that a binding MQS would exist (Ronnen, 1991).

As long as we stick to the assumption of full market coverage, and given that $\widehat{q}_{L}$ is in fact not subject to MQS regulation, then there exists no direct correlation between MQS regulation and the high-quality firm's incentive to adopt a new technology characterised by a higher $\eta$. With direct reference 
to Maxwell's analysis (1998, p. 357), eq. (10) never holds as $q_{L}=0$ is not admissible, and consequently the effect of a variation of $\eta$ on firm $H$ 's profits is always assessed as in eq. (11).

\section{Concluding remarks}

Reassessing Maxwell's (1998) analysis, we have shown that an MQS cannot be binding under full market coverage and therefore cannot influence the incentive to innovate by the high-quality firm. This conclusion raises a further issue, which we leave for future research: under what conditions regarding consumers' willingness to pay for quality may the introduction of an MQS induce a switch from full to partial coverage? This is a relevant question for the optimal design of this regulatory instrument that, so far, has remained neglected. 


\section{References}

[1] Gabszewicz, J.J. and J.-F. Thisse (1979), Price Competition, Quality and Income Disparities, Journal of Economic Theory, 20, 340-359.

[2] Garella, P.G. (2006), "Innocuous" Minimum Quality Standards, Economics Letters, 92, 368-374.

[3] Maxwell, J. W. (1998), Minimum Quality Standards as a Barrier to Innovation, Economics Letters, 58, 355-360.

[4] Ronnen, U. (1991), Minimum Quality Standards, Fixed Costs, and Competition, RAND Journal of Economics, 22, 490-504. 